\title{
OBSTRUCTIONS TO ALGEBRAIZING TOPOLOGICAL VECTOR BUNDLES
}

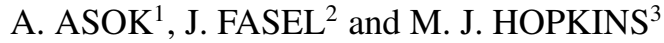 \\ ${ }^{1}$ Department of Mathematics, University of Southern California, 3620 S. Vermont Ave., \\ Los Angeles, CA 90089-2532, USA; \\ email: asok@usc.edu \\ ${ }^{2}$ Institut Fourier - UMR 5582, Université Grenoble Alpes CS 40700, \\ 38058 Grenoble Cedex 09, France; \\ email: jean.fasel@gmail.com \\ ${ }^{3}$ Department of Mathematics, Harvard University, One Oxford Street, \\ Cambridge, MA 02138, USA; \\ email:mjh@math.harvard.edu
}

Received 12 October 2015; accepted 16 July 2018

\begin{abstract}
Suppose $X$ is a smooth complex algebraic variety. A necessary condition for a complex topological vector bundle on $X$ (viewed as a complex manifold) to be algebraic is that all Chern classes must be algebraic cohomology classes, that is, lie in the image of the cycle class map. We analyze the question of whether algebraicity of Chern classes is sufficient to guarantee algebraizability of complex topological vector bundles. For affine varieties of dimension $\leqslant 3$, it is known that algebraicity of Chern classes of a vector bundle guarantees algebraizability of the vector bundle. In contrast, we show in dimension $\geqslant 4$ that algebraicity of Chern classes is insufficient to guarantee algebraizability of vector bundles. To do this, we construct a new obstruction to algebraizability using Steenrod operations on Chow groups. By means of an explicit example, we observe that our obstruction is nontrivial in general.
\end{abstract}

2010 Mathematics Subject Classification: 14F42 (primary); 32L05, 55R25, 13C10 (secondary)

\section{Introduction}

Suppose $X$ is a smooth complex algebraic variety. We write $X^{\text {an }}$ for $X(\mathbb{C})$ viewed as a complex manifold. Write $\mathscr{V}_{n}(X)$ for the set of isomorphism classes of rank- $n$ algebraic vector bundles on $X, \mathscr{V}^{\text {an }}(X)$ for the set of isomorphism classes of rank-

(C) The Author(s) 2019. This is an Open Access article, distributed under the terms of the Creative Commons Attribution licence (http://creativecommons.org/licenses/by/4.0/), which permits unrestricted re-use, distribution, and reproduction in any medium, provided the original work is properly cited. 
$n$ analytic vector bundles on $X^{\text {an }}$ and $\mathscr{V}^{\text {top }}(X)$ for the set of isomorphism classes of rank- $n$ complex topological vector bundles on $X^{\text {an }}$.

For any integer $n \geqslant 0$, the assignment $X \mapsto X^{\text {an }}$ gives rise to a sequence of functions

$$
\mathscr{V}_{n}(X) \longrightarrow \mathscr{V}^{\mathrm{an}}(X) \longrightarrow \mathscr{V}^{\text {top }}(X)
$$

An element of $\mathscr{V}^{\text {top }}(X)$ that lies in the image of the composite map $\mathscr{V}_{n}(X) \longrightarrow \mathscr{V}^{\text {top }}(X)$ will be called an algebraizable vector bundle. The motivating problem of this paper is as follows: characterize algebraizable vector bundles among topological vector bundles. This problem is very old; it is studied explicitly for projective varieties of small dimension, for example, in the work of Schwarzenberger [Sch61], Atiyah-Rees [AR76] and Bănică-Putinar [BP87]. Very little is known about this problem for varieties of dimension $\geqslant 4$.

Suppose $\mathcal{E}^{\text {top }} \rightarrow X^{\text {an }}$ is a complex topological vector bundle. If $\mathcal{E}^{\text {top }}$ is algebraizable, then the Chern classes $c_{i}^{\text {top }}\left(\mathcal{E}^{\text {top }}\right) \in H^{2 i}\left(X^{\text {an }}, \mathbb{Z}\right)$ of $\mathcal{E}^{\text {top }}$ are algebraic, that is, they lie in the image of the cycle class map

$$
c l: C H^{i}(X) \longrightarrow H^{2 i}\left(X^{\text {an }}, \mathbb{Z}\right) .
$$

Using this observation, one can show that the map $\mathscr{V}_{n}(X) \longrightarrow \mathscr{V}^{\text {top }}(X)$ is, in general, neither injective nor surjective. If $X$ is a smooth affine variety, then by Grauert's Oka principle (see [Gra58, Section 2 Satz I, II] or [For11, Theorem 7.2.1] for a textbook treatment), every topological vector bundle on $X^{\text {an }}$ admits a unique analytic structure, that is, the map $\mathscr{V}^{\text {an }}(X) \rightarrow \mathscr{V}^{\text {top }}(X)$ is a bijection. The following question is a concrete form of the problem stated above.

QUESTION 1. If $X$ is a smooth complex affine variety, and $\mathcal{E}^{\text {an }} \rightarrow X^{\text {an }}$ is a complex analytic vector bundle with algebraic Chern classes, then is $\mathcal{E}^{\text {an }}$ algebraizable?

Question 1 has a positive answer in small dimensions. Serre's splitting theorem [Ser58, Théorème 1] implies that any algebraic vector bundle of rank $r>\operatorname{dim} X$ on a smooth affine variety can be written as the direct sum of a vector bundle of rank $\leqslant \operatorname{dim} X$ and a trivial bundle (note: the smoothness hypothesis is unnecessary to apply Serre's result). To answer Question 1, it therefore suffices to establish that topological vector bundles of rank below the dimension with algebraic Chern classes are algebraizable.

For vector bundles of rank one on smooth affine varieties of any dimension, since $C H^{1}(X)=\operatorname{Pic}(X)$ algebraicity of the Chern classes essentially by definition guarantees algebraizability; it follows immediately that Question 1 has a positive answer for varieties of dimension one. In dimension two, a positive answer to 
Question 1 follows from the work of Murthy and Swan [MS76] who show that if $X$ is a smooth complex affine surface, then for any pair $\left(c_{1}, c_{2}\right) \in C H^{1}(X) \times$ $\mathrm{CH}^{2}(\mathrm{X})$ there is an algebraic vector bundle of rank two with those Chern classes. Similarly, in dimension three, a positive answer to Question 1 follows from the work of Kumar and Murthy [KM82, Theorem 2.1]. In particular, Kumar and Murthy established existence of algebraic vector bundles of rank $\leqslant 3$ with arbitrary prescribed Chern classes on smooth affine 3-folds.

The main result of this paper is that Question 1 admits a negative answer in the first unknown case: rank-two vector bundles on smooth complex affine varieties of dimension $\geqslant 4$. To see this, we will give a necessary and sufficient condition for algebraizability of rank-two bundles on smooth complex affine 4-folds involving the integral Steenrod squaring operation $S q^{2}$ on Chow groups; this operation was described by Voevodsky [Voe03] and Brosnan [Bro03] though for our purposes the latter (more elementary) description is sufficient. We then show, by means of explicit examples, that the necessary and sufficient condition for algebraizability we write down is not always satisfied. More precisely, we establish the following results (the first result is established just after Theorem 2.2.2 in the body of the text).

THEOREM 2. Suppose $X$ is a smooth complex affine variety of dimension four, and $\mathcal{E}^{\text {an }} \rightarrow X^{\text {an }}$ is a rank-two complex analytic vector bundle with Chern classes $c_{i}^{\text {top }} \in H^{2 i}\left(X^{\text {an }}, \mathbb{Z}\right)$. Assume the Chern classes $c_{i}^{\text {top }}$ of $\mathcal{E}^{\text {an }}$ are algebraic, that is, lie in the image of the cycle class map cl. The bundle $\mathcal{E}^{\text {an }}$ is algebraizable if and only if we may find $\left(c_{1}, c_{2}\right) \in \mathrm{CH}^{1}(X) \times \mathrm{CH}^{2}(X)$ with $\left(\mathrm{cl}\left(c_{1}\right), \mathrm{cl}\left(c_{2}\right)\right)=\left(c_{1}^{\text {top }}, c_{2}^{\text {top }}\right)$ such that $S q^{2} c_{2}+c_{1} \cup c_{2}=0 \in C H^{3}(X) / 2$.

REMARK 3. Note that, even if $c_{1}^{\text {top }}$ is zero in the above statement, unless the cycle class map $c l: C H^{1}(X) \rightarrow H^{2}(X, \mathbb{Z})$ is injective in degree one we cannot guarantee that $c_{1}$ can be chosen to vanish. A priori it is possible that $S q^{2} c_{2}+c_{1} \cup c_{2}$ is always zero, but the following result shows that this is not the case.

THEOREM 4 (See Corollary 3.1.5). There exists a smooth hypersurface $Z$ of bidegree $(3,4)$ in $\mathbb{P}^{1} \times \mathbb{P}^{3}$, such that, setting $X:=\left(\mathbb{P}^{1} \times \mathbb{P}^{3}\right) \backslash Z$, the following statements hold:

(a) The cycle class map cl: $\mathrm{CH}^{i}(X) \rightarrow H^{2 i}\left(X^{\mathrm{an}}, \mathbb{Z}\right)$ is injective for $i \leqslant 2$.

(b) The manifold $X^{\text {an }}$ carries a rank-two topological vector bundle $\mathcal{E}^{\text {an }}$ with Chern classes $\left(0, c_{2}^{\text {top }}\right)$ such that $c_{2}^{\text {top }}$ is algebraic and the unique lift $c_{2}$ of $c_{2}^{\text {top }}$ granted by (a) satisfies $S q^{2} c_{2} \neq 0$ (in particular, $\mathcal{E}^{\text {an }}$ is not algebraizable). 
Theorem 2 is established by first observing that the map $\mathscr{V}_{n}(X) \rightarrow \mathscr{V}_{n}^{\text {top }}(X)$ factors through $\left[X, \mathrm{BGL}_{n}\right]_{\mathbb{A}^{1}}$, that is, the set of $\mathbb{A}^{1}$-homotopy classes of maps $X$ to $\mathrm{BGL}_{n}$ (the set $\left[X, \mathrm{BGL}_{n}\right]_{\mathbb{A}^{1}}$ has been called the set of motivic vector bundles on $X$ by the authors). Thus, to produce the obstruction, it suffices to obstruct existence of an $\mathbb{A}^{1}$-homotopy class of maps, that is, to obstruct existence of a 'motivic' lift of a given homotopy class. This is accomplished by analysis of the Moore-Postnikov factorization of the map assigning to the universal rank-two vector bundle over the Grassmannian its Chern classes; these ideas are discussed in Section 2.1. The primary obstruction to the existence of a lifting yields the condition of the statement, and we establish a vanishing theorem showing that, under suitable hypotheses, all higher obstructions vanish. To prove necessity of the vanishing of the obstruction, we appeal to Morel's vector bundle classification: if $X$ is smooth and affine, then $\left[X, \mathrm{BGL}_{n}\right]_{\mathbb{A}^{1}} \cong \mathscr{V}_{n}(X)$ (see [AHW17, Theorem 1]).

The construction of the example in Theorem 4 is closely related with the failure of the integral Hodge conjecture for the hypersurface $Z$. The failure of injectivity of the cycle class map $C H^{3}(X) \rightarrow H^{6}\left(X^{\text {an }}, \mathbb{Z}\right)$ is precisely what allows the examples above to exist. In Section 3.2, we explain how some general conjectures on algebraic cycles suggest examples like those above are 'generic'. By considering products of the form $X \times \mathbb{A}^{n}$ with $X$ as in Theorem 4, one may construct examples of nonalgebraizable topological vector bundles with algebraic Chern classes in any dimension $\geqslant 4$.

REMARK 5. Theorem 4 also provides a counterexample to a related K-theoretic variant of Question 1. Indeed, for any smooth $\mathbb{C}$-scheme $X$, we may consider the Grothendieck groups $K_{0}(X)$ and $K_{0}^{\text {top }}\left(X^{\text {an }}\right)$. The functions $\mathscr{V}_{n}(X) \rightarrow \mathscr{V}_{n}^{\text {top }}\left(X^{\text {an }}\right)$ (for varying $n$ ) induce a function $K_{0}(X) \rightarrow K_{0}^{\text {top }}(X)$; we will say that a class in $K_{0}^{\text {top }}(X)$ is algebraic if it lies in the image of this map. One might ask whether topological vector bundles whose associated K-theory class is algebraic themselves admit algebraic structures.

Since Chern classes factor through K-theory, algebraicity of the topological Ktheory class of a vector bundle is a stronger restriction than algebraicity of Chern classes. [KM82, Theorem 2.1] essentially shows that the K-theoretic variant of Question 1 admits a positive solution for smooth affine $\mathbb{C}$-schemes of dimension $\leqslant 3$. On the other hand, the proof of Proposition 2.2.1 shows that the restriction on Chern classes appearing in Theorem 2 is precisely the primary obstruction to building a rank-two vector bundle on a smooth affine 4-fold given a fixed class in $K_{0}(X)$. Thus in dimension $\geqslant 4$, the K-theoretic variant of Question 1 mentioned in the previous paragraph also admits a negative solution. 


\section{Obstruction theory: proof of Theorem 2}

The goal of this section is to prove Theorem 2. We begin with some preliminaries regarding Chern classes and obstruction theory in $\mathbb{A}^{1}$-homotopy theory. We will use rather freely the terminology of [MV99, Mor12]; instead of bulking up this paper with a long section of notation and terminology, we have chosen to focus on the argument; we will follow the notations and conventions of [AF15b, Section 2.1] and we refer the reader there for terminology not explicitly defined here (for example, spaces, homotopy sheaves, strong and strict $\mathbb{A}^{1}$ invariance of sheaves and their basic properties). When we consider cohomology of a sheaf on a smooth scheme, we mean cohomology in the Nisnevich site. We also remind the reader that strongly or strictly $\mathbb{A}^{1}$-invariant sheaves are unramified and that to check a morphism of unramified sheaves is an isomorphism, it suffices to check this on sections over finitely generated extensions of the base field. For a general discussion of the Moore-Postnikov factorization in $\mathbb{A}^{1}$-homotopy theory, we refer the reader to [AF15b, Section 6].

\subsection{Chern classes and the basic obstruction theory problem. Write} $\mathrm{BGL}_{n}$ for the simplicial classifying space of $\mathrm{GL}_{n}$ (see, for example, [MV99, Section 4.1]). The determinant map $\mathrm{GL}_{n} \rightarrow \mathbf{G}_{m}$ induces a map of classifying spaces $\mathrm{BGL}_{n} \rightarrow B \mathbf{G}_{m}$. If $X \rightarrow \mathrm{BGL}_{n}$ is a simplicial homotopy class of maps representing a rank- $n$ vector bundle on a smooth scheme $X$, then the composite map $X \rightarrow \mathrm{BGL}_{n} \rightarrow B \mathbf{G}_{m}$ represents the determinant line bundle of this vector bundle. Some $\mathbb{A}^{1}$-homotopy sheaves of $\mathrm{BGL}_{n}$ were computed in [Mor12, Theorem 7.20]; we refer the reader to [AF14a, Lemma 3.1, Theorem 3.2] for convenient references in the form we require.

LEMMA 2.1.1. There are canonical isomorphisms $\boldsymbol{\pi}_{1}^{\mathbb{A}^{1}}\left(\mathrm{BGL}_{n}\right) \stackrel{\sim}{\rightarrow} \mathbf{G}_{m}$ (induced by the determinant homomorphism) and $\boldsymbol{\pi}_{2}^{\mathbb{A}^{1}}\left(\mathrm{BGL}_{2}\right) \cong \mathbf{K}_{2}^{M W}$.

The motivic cohomology of $\mathrm{BGL}_{n}$ is a polynomial algebra over the motivic cohomology of a point in variables $c_{1}, \ldots, c_{n}$ where $c_{i}$ has bidegree $(2 i$, $i$ ) (this is 'well known', but see, for example, [Pus04, Proposition 2] for a precise statement; Pushin's argument is a version of the argument of [Gil81], which itself goes back to Grothendieck's axiomatic treatment of Chern classes). By Voevodsky's (unstable) $\mathbb{A}^{1}$-representability of motivic cohomology [Del09, Section 2.3 Theorem 2], each $c_{i}$ corresponds to an $\mathbb{A}^{1}$-homotopy class of maps

$$
c_{i}: \mathrm{BGL}_{n} \rightarrow K(\mathbb{Z}(i), 2 i) .
$$


There is an $\mathbb{A}^{1}$-weak equivalence $\mathrm{Gr}_{n} \rightarrow \mathrm{BGL}_{n}$, where $\mathrm{Gr}_{n}$ is the infinite Grassmannian [MV99, Section 4 Proposition 3.7]. Here and henceforth, we can fix suitable $\mathbb{A}^{1}$-fibrant models of $\mathrm{BGL}_{n}$ and $K(\mathbb{Z}(i), 2 i)$ so that the $\mathbb{A}^{1}$-homotopy classes of maps above are represented by actual morphisms of spaces $\mathrm{Gr}_{n} \rightarrow$ $K(\mathbb{Z}(i), 2 i)$.

Morel and Voevodsky introduced a complex realization functor [MV99, Section 3.3]. Under complex realization, $\mathrm{Gr}_{n}$ is sent to the usual complex Grassmannian. The model of $K(\mathbb{Z}(i), 2 i)$ in terms of effective cycles [Voe04, Section 6] and the classical Dold-Thom theorem show that the complex realization of $K(\mathbb{Z}(i), 2 i)$ is $K(\mathbb{Z}, 2 i)$. Moreover, the maps $c_{i}: \mathrm{Gr}_{n} \rightarrow K(\mathbb{Z}(i)$, $2 i)$ are sent by realization to the usual Chern class maps $c_{i}^{\text {top }}: \mathrm{Gr}_{n} \rightarrow K(\mathbb{Z}, 2 i)$. Indeed, this observation is a consequence of (1) the fact that the finite-dimensional Grassmannian varieties $\mathrm{Gr}_{n, N}$ admit a cellular decomposition [Ful98, Example 1.9.1] and thus the cycle class map from Chow groups to ordinary cohomology is an isomorphism (see also [DI05, Proposition 4.4] for a more homotopic statement), (2) the fact that $\mathrm{Gr}_{n}$ is a filtered colimit of the finite-dimensional Grassmannian varieties $\mathrm{Gr}_{n, N}$ by construction, and (3) the fact that motivic cohomology of $\mathrm{Gr}_{n, N}$ in any given degree stabilizes for $N$ large enough, for example, by Totaro's argument [EG98, Definition-Proposition 1 and Section 2.7]. We use the compatibilities discussed above without mention in the following.

The space $K(\mathbb{Z}(n), 2 n)$ is not an Eilenberg-Mac Lane space in the sense that its $\mathbb{A}^{1}$-homotopy sheaves are not, in general, concentrated in a single degree. Nevertheless, one may identify the $\mathbb{A}^{1}$-homotopy sheaves of $K(\mathbb{Z}(n), 2 n)$. The first statement is simply a reformulation of the $\mathbb{A}^{1}$-representability of motivic cohomology mentioned above, while the second statement is a reformulation of the Nesterenko-Suslin-Totaro theorem (see, for example, [MVW06, Theorem 5.1]).

LEMMA 2.1.2. There is a canonical isomorphism $\pi_{i}^{\mathbb{A}^{1}}(K(\mathbb{Z}(n), 2 n)) \cong \mathbf{H}^{2 n-i, n}$, where $\mathbf{H}^{2 n-i, n}$ is the sheafification of the presheaf $U \mapsto H^{2 n-i, n}(U, \mathbb{Z})$. In particular, $K(\mathbb{Z}(n), 2 n)$ is $\mathbb{A}^{1}-(n-1)$-connected and its first nonvanishing $\mathbb{A}^{1}$ homotopy sheaf is $\mathbf{K}_{n}^{M}$, the nth unramified Milnor K-theory sheaf.

Ideally, we would study the existence of a vector bundle of rank two with given Chern classes $\left(c_{1}, c_{2}\right)$ by studying an obstruction theory problem deduced from the Moore-Postnikov factorization of the map $\left(c_{1}, c_{2}\right): \mathrm{BGL}_{2} \rightarrow K(\mathbb{Z}(1)$, $2) \times K(\mathbb{Z}(2), 4)$. The fact that $K(\mathbb{Z}(n), 2 n)$ is not an Eilenberg-Mac Lane space causes various technical complications and we use the description of its homotopy sheaves provided above to produce an equivalent yet technically simpler problem. 
The first stage of the $\mathbb{A}^{1}$-Postnikov tower of $K(\mathbb{Z}(n), 2 n)$ yields, by appeal to Lemma 2.1.2, a canonical map $K(\mathbb{Z}(n), 2 n) \rightarrow K\left(\mathbf{K}_{n}^{M}, n\right)$. In particular, composition of the universal $n$th Chern class with this map yields a map:

$$
c_{n}^{\prime}: \mathrm{BGL}_{r} \rightarrow K\left(\mathbf{K}_{n}^{M}, n\right)
$$

for any $r \geqslant n$; this is a modified version of the universal $n$th Chern class.

REMARK 2.1.3. Since $\mathbf{K}_{1}^{M}=\mathbf{G}_{m}$ and since the motivic complex $\mathbb{Z}(1)$ is $\mathbf{G}_{m}$ concentrated in a single degree [MVW06, Theorem 4.1], the map $K(\mathbb{Z}(1), 2) \rightarrow$ $K\left(\mathbf{K}_{1}^{M}, 1\right)$ induced by the Postnikov tower is a simplicial weak equivalence. In particular, the maps $c_{1}$ and $c_{1}^{\prime}$ lie in the same simplicial homotopy class. Explicitly identifying the homotopy sheaves of $\pi_{i}^{\mathbb{A}^{1}}(K(\mathbb{Z}(n), 2 n))$ in all degrees seems at the moment intractable: the Beilinson-Soule vanishing conjecture predicts that $K(\mathbb{Z}(n), 2 n)$ is $\mathbb{A}^{1}-(2 n-1)$-truncated, that is, its homotopy sheaves vanish for $i>2 n-1$.

2.2. Homotopy sheaves and Moore-Postnikov factorizations. Consider the map

$$
\left(c_{1}^{\prime}, c_{2}^{\prime}\right): \mathrm{BGL}_{2} \rightarrow K\left(\mathbf{K}_{1}^{M}, 1\right) \times K\left(\mathbf{K}_{2}^{M}, 2\right),
$$

where $c_{1}^{\prime}$ and $c_{2}^{\prime}$ are the maps mentioned in (2.1). Our goal is to analyze the homotopy fiber of this map. Write $\mathscr{F}_{2}$ for the $\mathbb{A}^{1}$-homotopy fiber of $\left(c_{1}^{\prime}, c_{2}^{\prime}\right)$.

By Lemma 2.1.1 there is a canonical identification $\pi_{1}^{\mathbb{A}^{1}}\left(\mathrm{BGL}_{2}\right) \cong \mathbf{G}_{m}$ and the map $c_{1}^{\prime}$ yields an isomorphism of $\mathbb{A}^{1}$-fundamental sheaves of groups (see also Remark 2.1.3). As a consequence, we will be considering a twisted obstruction theory problem and we will have to keep track of the action of $\mathbf{G}_{m}$ on the higher $\mathbb{A}^{1}$-homotopy sheaves of the $\mathbb{A}^{1}$-homotopy fiber. We begin by identifying the relevant $\mathbb{A}^{1}$-homotopy sheaves of $\mathscr{F}_{2}$ together with the action of $\mathbf{G}_{m}$ (for the relevant definitions about twisted $\mathbb{A}^{1}$-homotopy sheaves, we refer the reader to [AF15b, Section 2.4]).

PROPOSITION 2.2.1. There are canonical isomorphisms $\pi_{2}^{\mathbb{A}^{1}}\left(\mathscr{F}_{2}\right) \cong \mathbf{I}^{3}$ and $\pi_{3}^{\mathbb{A}^{1}}\left(\mathscr{F}_{2}\right) \cong \pi_{3}^{\mathbb{A}^{1}}\left(\mathrm{BGL}_{2}\right) \cong \boldsymbol{\pi}_{2}^{\mathbb{A}^{1}}\left(\mathrm{SL}_{2}\right)$. Moreover, the actions of $\mathbf{G}_{m}$ on these sheaves coincide with the actions described in [AF14b, Propositions 6.3 and 6.5].

Proof. Observe that $K\left(\mathbf{K}_{1}^{M}, 1\right) \times K\left(\mathbf{K}_{2}^{M}, 2\right)$ is $\mathbb{A}^{1}$-2-truncated, that is, has $\mathbb{A}^{1}$ homotopy sheaves concentrated in degrees $\leqslant 2$. The second assertion is therefore immediate from the isomorphism $\pi_{3}^{\mathbb{A}^{1}}\left(\mathrm{BGL}_{2}\right) \cong \pi_{2}^{\mathbb{A}^{1}}\left(\mathrm{SL}_{2}\right)$. 
For the first statement, the long exact sequence in $\mathbb{A}^{1}$-homotopy sheaves of a fibration yields (using Lemma 2.1.1) a short exact sequence

$$
0 \longrightarrow \pi_{2}^{\mathbb{A}^{1}}\left(\mathscr{F}_{2}\right) \longrightarrow \mathbf{K}_{2}^{M W} \longrightarrow \mathbf{K}_{2}^{M} \longrightarrow 0 .
$$

On the other hand, recall that there is an exact sequence of sheaves of the form

$$
0 \longrightarrow \mathbf{I}^{3} \longrightarrow \mathbf{K}_{2}^{M W} \longrightarrow \mathbf{K}_{2}^{M} \longrightarrow 0
$$

this is the sheafified version of the exact sequence of [Mor04, Corollaire 5.4]. We will identify these two exact sequences.

To this end, let us unwind some of the identifications. Recall that the $\mathbb{A}^{1}$-fiber sequence

$$
\mathbf{G}_{m} \longrightarrow \mathrm{BSL}_{2} \longrightarrow \mathrm{BGL}_{2}
$$

yields the identification $\boldsymbol{\pi}_{2}^{\mathbb{A}^{1}}\left(\mathrm{BSL}_{2}\right) \cong \boldsymbol{\pi}_{2}^{\mathbb{A}^{1}}\left(\mathrm{BGL}_{2}\right)$.

Identify $\mathrm{SL}_{2} \cong \mathrm{Sp}_{2}$ and consider the following commutative diagram:

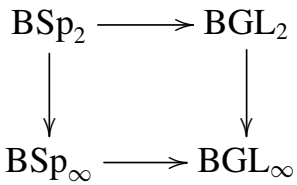

The left vertical map induces an isomorphism on $\pi_{2}^{\mathbb{A}^{1}}$ by [AF14b, Theorem 2.6] and yields the isomorphism $\boldsymbol{\pi}_{2}^{\mathbb{A}^{1}}\left(\mathrm{BSp}_{2}\right) \cong \boldsymbol{\pi}_{2}^{\mathbb{A}^{1}}\left(\mathrm{BSp}_{\infty}\right) \cong \mathbf{K S \mathbf { p } _ { 2 }}$. By Suslin's theorem, $\mathbf{K S p} \mathbf{p}_{2} \cong \mathbf{K}_{2}^{M W}$ [Sus87, Corollaries 6.2, 6.4 and Theorem 6.5] (see [AF17, Theorem 4.1.2] and the discussion there for an explanation in the context we consider). On the other hand, the map on $\pi_{2}^{\mathbb{A}^{1}}$ induced by the right vertical map is a map $\mathbf{K}_{2}^{M W} \rightarrow \mathbf{K}_{2}^{M}$ (see [AF14a, Lemma 3.1 and Theorem 3.2]). The bottom horizontal map induces the forgetful map, which coincides with the standard surjection $\mathbf{K}_{2}^{M W} \rightarrow \mathbf{K}_{2}^{M}$.

The second Chern class map $\mathrm{BGL}_{2} \rightarrow K\left(\mathbf{K}_{2}^{M}, 2\right)$ necessarily factors through the map $\mathrm{BGL}_{2} \rightarrow \mathrm{BGL}_{\infty}$ and the map $\mathrm{BGL}_{\infty} \rightarrow K\left(\mathbf{K}_{2}^{M}, 2\right)$ is an isomorphism on $\pi_{2}^{\mathbb{A}^{1}}$ by Matsumoto's theorem identifying Quillen's $K_{2}$ of a field with Milnor's $K_{2}$. Therefore, the second Chern class map induces a map on $\pi_{2}^{\mathbb{A}^{1}}$ that, up to the identifications described above, coincides with the forgetful map $\mathbf{K}_{2}^{M W} \rightarrow \mathbf{K}_{2}^{M}$, which is precisely what is asserted above.

By construction, the actions mentioned in the statement are inherited from the $\mathbf{G}_{m}$-actions on homotopy sheaves of $\mathrm{BGL}_{2}$ as described in the referenced propositions.

THEOREM 2.2.2. Suppose $X$ is a smooth affine 4-fold over an algebraically closed field having characteristic unequal to 2 , and fix a line bundle $\mathcal{L}$ on $X$ 
with first Chern class $c_{1} \in C H^{1}(X)$. Given $c_{2} \in C H^{2}(X)$, the pair $\left(c_{1}, c_{2}\right)$ are the first and second Chern classes of a vector bundle of rank two and determinant $\mathcal{L}$ if and only if $S q^{2} c_{2}+c_{1} \cup c_{2}=0$.

Proof. Suppose $X$ is a smooth affine 4-fold over $\operatorname{Spec} k$ and fix $\left(c_{1}, c_{2}\right) \in$ $\mathrm{CH}^{1}(\mathrm{X}) \times \mathrm{CH}^{2}(\mathrm{X})$ as in the statement. Such a pair determines a map $\psi: X \rightarrow$ $K\left(\mathbf{K}_{1}^{M}, 1\right) \times \mathbf{K}\left(\mathbf{K}_{2}^{M}, 2\right)$. Fix a line bundle $\mathcal{L}$ on $X$ representing $c_{1}$. We now analyze the Moore-Postnikov factorization of the map $\left(c_{1}, c_{2}^{\prime}\right): \mathrm{BGL}_{2} \rightarrow K\left(\mathbf{K}_{1}^{M}, 1\right)$ $\times K\left(\mathbf{K}_{2}^{M}, 2\right)$.

This analysis proceeds in several steps, which we now outline. First, we analyze the primary obstruction: we begin by constructing a 'quotient' of the primary obstruction, which is easier to identify. Then, we show that this 'quotient' of the primary obstruction actually coincides with the primary obstruction by means of suitable cohomological vanishing statements. Next, we analyze the secondary obstruction. We show the secondary obstruction vanishes again by establishing a general cohomological vanishing result. Finally, we appeal to general theory to show that no further obstructions can arise.

Step 1: Analyzing the primary obstruction. The primary obstruction to lifting $\psi$ to a map $X \rightarrow \mathrm{BGL}_{2}$ is, by means of Proposition 2.2.1, a class in $H^{3}\left(X, \mathbf{I}^{3}(\mathcal{L})\right)$.

Step 1a: A quotient of the primary obstruction. To identify this obstruction more explicitly, we consider the exact sequence of [Fas13, Section 2.1, p. 423]:

$$
0 \longrightarrow \mathbf{I}^{j+1}(\mathcal{L}) \longrightarrow \mathbf{I}^{j}(\mathcal{L}) \longrightarrow \mathbf{I}^{j} / \mathbf{I}^{j+1} \longrightarrow 0 .
$$

By the sheafified version of the Milnor conjecture on quadratic forms [OVV07] there is a canonical isomorphism of sheaves $\mathbf{I}^{j} / \mathbf{I}^{j+1} \cong \mathbf{K}_{j}^{M} / 2$. We consider the composite map (via this isomorphism)

$$
H^{2}\left(X, \mathbf{K}_{2}^{M}\right) \longrightarrow H^{3}\left(X, \mathbf{I}^{3}(\mathcal{L})\right) \longrightarrow H^{3}\left(X, \mathbf{K}_{3}^{M} / 2\right) .
$$

The first map here is precisely the $k$-invariant in the Moore-Postnikov factorization and the identifications of Proposition 2.2.1 show that it is exactly the connecting homomorphism in the long exact sequence in cohomology associated with the exact sequence of sheaves on $X$ :

$$
0 \longrightarrow \mathbf{I}^{3}(\mathcal{L}) \longrightarrow \mathbf{K}_{2}^{M W}(\mathcal{L}) \longrightarrow \mathbf{K}_{2}^{M} \longrightarrow 0 .
$$

The composite above can be described in a fashion that extends a result of Totaro. More precisely, we claim that if $c_{2} \in H^{2}\left(X, \mathbf{K}_{2}^{M}\right)$, then the map just described,

$$
H^{2}\left(X, \mathbf{K}_{2}^{M}\right) \longrightarrow H^{3}\left(X, \mathbf{K}_{3}^{M} / 2\right),
$$


sends $c_{2} \mapsto S q^{2} c_{2}+c_{1}(\mathcal{L}) \cup c_{2}$. First, we treat the case where $\mathcal{L}$ is trivial. In that case, the map $H^{2}\left(X, \mathbf{K}_{2}^{M}\right) \longrightarrow H^{3}\left(X, \mathbf{K}_{3}^{M} / 2\right)$ factors through the mod 2 reduction map $C H^{2}(X)=H^{2}\left(X, \mathbf{K}_{2}^{M}\right) \rightarrow H^{2}\left(X, \mathbf{K}_{2}^{M} / 2\right)=C H^{2}(X) / 2$ (see [AF15a, Diagram 2.1.2] and the preceding discussion). Therefore, [Tot03, Theorem 1.1] implies that the composite map is precisely the composite of the mod 2 reduction map and $S q^{2}$, that is, the integral Steenrod squaring map. To treat the general case, we appeal to [AF15a, Theorem 3.4.1] (applied in the case $i=j=2$ ), which reduces the description of the relevant operation when $\mathcal{L}$ is nontrivial to the case where $\mathcal{L}$ is trivial.

Step 1b: The actual primary obstruction. The relationship between the obstruction just mentioned and the actual primary obstruction is measured by the difference between the groups $H^{3}\left(X, \mathbf{I}^{3}(\mathcal{L})\right)$ and $H^{3}\left(X, \mathbf{K}_{3}^{M} / 2\right)$. In this case, we have the long exact sequence

$$
\begin{aligned}
\cdots & \longrightarrow H^{3}\left(X, \mathbf{I}^{4}(\mathcal{L})\right) \longrightarrow H^{3}\left(X, \mathbf{I}^{3}(\mathcal{L})\right) \longrightarrow H^{3}\left(X, \mathbf{K}_{3}^{M} / 2\right) \\
& \longrightarrow H^{4}\left(X, \mathbf{I}^{4}(\mathcal{L})\right) \longrightarrow \cdots .
\end{aligned}
$$

Since $X$ is a smooth affine 4-fold over an algebraically closed field, by [AF14b, Proposition 5.2] the groups $H^{3}\left(X, \mathbf{I}^{4}(\mathcal{L})\right)$ and $H^{4}\left(X, \mathbf{I}^{4}(\mathcal{L})\right)$ vanish. Thus, the primary obstruction coincides with the 'quotient' constructed in Step 1a, that is, the primary obstruction to lifting is precisely the vanishing of $S q^{2} c_{2}+c_{1} \cup c_{2}$.

Step 2: Analyzing the secondary obstruction. If the primary obstruction vanishes, then we can choose a lift to the second stage of the Moore-Postnikov factorization of the map $\left(c_{1}, c_{2}^{\prime}\right)$. Upon choosing a lift to this stage, we obtain a secondary obstruction lying in $H^{4}\left(X, \pi_{3}^{\mathbb{A}^{1}}\left(\mathscr{F}_{2}\right)(\mathcal{L})\right)$. By means of Proposition 2.2.1, this obstruction is an element of $H^{4}\left(X, \pi_{3}^{\mathbb{A}^{1}}\left(\mathrm{BGL}_{2}\right)(\mathcal{L})\right)$. A priori, this obstruction depends on the choice of lift, but we claim $H^{4}(X$, $\left.\pi_{3}^{\mathbb{A}^{1}}\left(\mathrm{BGL}_{2}\right)(\mathcal{L})\right)=0$, independent of this choice.

To see this, recall by [AF14b, Theorem 3.3] that $\boldsymbol{\pi}_{3}^{\mathbb{A}^{1}}\left(\mathrm{BSp}_{2}\right)$ is an extension of $\mathbf{K}_{3}^{\mathrm{Sp}}=\mathbf{G W}_{3}^{2}$ by a certain sheaf $\mathbf{T}_{4}^{\prime}$. Observe first that $H^{4}\left(X, \mathbf{G W}_{3}^{2}(\mathcal{L})\right)=0$ by explicit construction of the Gersten resolution. Indeed, $H^{4}\left(X, \mathbf{G W}_{3}^{2}(\mathcal{L})\right)$ is simply a quotient of $\bigoplus_{x \in X^{(4)}}\left(\mathbf{G W}_{3}^{2}(\mathcal{L})\right)_{-4}\left(\kappa_{x}\right)$ and the latter vanishes by [AF14b, Lemma 4.11].

By [AF14b, Corollary 4.9] the induced $\mathbf{G}_{m}$-action on $\mathbf{T}_{4}^{\prime}$ is trivial. Again using [AF14b, Theorem 3.3], $\mathbf{S}_{4}^{\prime}$ is a quotient of $\mathbf{T}_{4}^{\prime}$ by $\mathbf{D}_{5}$; the sheaf $\mathbf{S}_{4}^{\prime}$ is itself a quotient of $\mathbf{K}_{4}^{M} / 12$ while the sheaf $\mathbf{D}_{5}$ is a quotient of $\mathbf{I}^{5}$. Observe that $H^{4}(X$, $\left.\mathbf{K}_{4}^{M} / 12\right) \cong C H^{4}(X) / 12$. Since $X$ is smooth and affine over an algebraically closed field, $\mathrm{CH}^{4}(X)$ is divisible and therefore, $H^{4}\left(X, \mathbf{K}_{4}^{M} / 12\right)$ is trivial. On the other hand, $H^{4}\left(X, \mathbf{I}^{5}\right)$ is trivial since [AF14b, Proposition 5.1] shows the sheaf $\mathbf{I}^{5}$ is itself trivial when restricted to $X$. 
Step 3. Lifting to $\mathrm{BGL}_{2}$. Since the secondary obstruction vanishes, we may choose a lift to the third stage of the Moore-Postnikov factorization of the map. Since $X$ has Nisnevich cohomological dimension four, there are no further obstructions to lifting and we may arbitrarily make a choice of lift of $X$ to the fourth stage of the Moore-Postnikov factorization of the map $\left(c_{1}, c_{2}^{\prime}\right)$. Moreover, again using the fact that $X$ has Nisnevich cohomological dimension four we see that beyond the fourth stage of the factorization all lifts are uniquely determined. Thus, by the same argument as in [AF14b, Proposition 6.2], we obtain an element of $\left[X, \mathrm{BGL}_{2}\right]_{\mathbb{A}^{1}}$. Combining the discussion of the previous steps we see that a necessary and sufficient condition to lift $\left(c_{1}, c_{2}\right) \in C H^{1}(X) \times C H^{2}(X)$ to an element of $\left[X, \mathrm{BGL}_{2}\right]_{\mathbb{A}^{1}}$ is the vanishing of $S q^{2} c_{2}+c_{1} \cup c_{2} \in C H^{3}(X) / 2$.

Step 4. Geometrization. Finally, we apply Morel's $\mathbb{A}^{1}$-representability theorem for vector bundles [AHW17, Theorem 1] to identify $\left[X, \mathrm{BGL}_{2}\right]_{\mathbb{A}^{1}}$ with $\mathscr{V}_{2}(X)$.

Proof of Theorem 2. Consider the map

$$
\left(c_{1}^{\text {top }}, c_{2}^{\text {top }}\right): \mathrm{BGL}_{2}(\mathbb{C}) \longrightarrow K(\mathbb{Z}, 2) \times K(\mathbb{Z}, 4) .
$$

The map $\left(c_{1}^{\text {top }}, c_{2}^{\text {top }}\right)$ is a 4-equivalence and thus the homotopy fiber is 5-connected. If $X$ is a smooth affine 4-fold, then $X^{\text {an }}$ has the homotopy type of a CW complex of dimension $\leqslant 4$. A straightforward obstruction theory argument then shows that there is a bijection $\mathscr{V}_{2}^{\text {top }}(X) \stackrel{\sim}{\rightarrow} H^{2}\left(X^{\text {an }}, \mathbb{Z}\right) \times H^{4}\left(X^{\text {an }}, \mathbb{Z}\right)$. Combining this observation with Theorem 2.2.2 yields Theorem 2 .

REMARK 2.2.3. It is possible to establish Theorem 2 by a direct analysis of the Moore-Postnikov factorization of $\left(c_{1}, c_{2}\right): \mathrm{BGL}_{2} \rightarrow K(\mathbb{Z}(1), 2) \times K(\mathbb{Z}(2), 4)$, but identifying the $\mathbb{A}^{1}$-homotopy sheaves of the $\mathbb{A}^{1}$-homotopy fiber is slightly more complicated than the approach described above.

\section{Constructing examples: proof of Theorem 4}

The goal of this section is to construct explicit examples of smooth complex affine varieties for which (a) the cycle class map $C H^{i}(X) \rightarrow H^{2 i}(X, \mathbb{Z})$ is injective for $i \leqslant 2$ and (b) there exist classes $\alpha \in C H^{2}(X)$ such that $S q^{2} \alpha \neq 0$.

3.1. Explicit examples of nonalgebraizable vector bundles. If $\operatorname{dim} X<3$, $C H^{3}(X)$ is trivial by definition and if $\operatorname{dim} X=3$ then $C H^{3}(X)$ is divisible (which is classical); thus, if $\operatorname{dim} X \leqslant 3$, then $C H^{3}(X) / 2$ is trivial. Therefore the first dimension that can support nontrivial examples of the kind we envision is dimension four. 
Proposition 3.1.1. Suppose $Y$ is a smooth projective variety of dimension $\geqslant 4$ and $Z$ is an ample hypersurface on $Y$ (that is, $\mathcal{O}_{Y}(Z)$ is an ample line bundle) and set $X:=Y \backslash Z$. If the cycle class map $C H^{i}(Y) \rightarrow H^{2 i}\left(Y^{\text {an }}, \mathbb{Z}\right)$ is an isomorphism for $i \leqslant 2$, then the cycle class maps $C^{i}(X) \rightarrow H^{2 i}\left(X^{\mathrm{an}}, \mathbb{Z}\right)$ are injective for $i \leqslant 2$.

Proof. Using the assumption on the dimension and the hypothesis that $Z$ is an ample hypersurface, the Grothendieck-Lefschetz theorem guarantees that the pullback map $\operatorname{Pic}(Y) \rightarrow \operatorname{Pic}(Z)$ is an isomorphism [Har70, Corollary IV.3.3]. By the usual Lefschetz hyperplane theorem [Voi07, Theorem 1.1], the map $H^{i}\left(Y^{\text {an }}\right.$, $\mathbb{Z}) \rightarrow H^{i}\left(Z^{\text {an }}, \mathbb{Z}\right)$ is an isomorphism for $i \leqslant 2$ and injective for $i=3$. Since the cycle class map commutes with pullbacks [Voi07, Proposition 9.2.1(i)] and since the map $\operatorname{Pic}(Y) \rightarrow H^{2}\left(Y^{\text {an }}, \mathbb{Z}\right)$ is an isomorphism by assumption, we conclude that $\operatorname{Pic}(Z) \rightarrow H^{2}\left(Z^{\text {an }}, \mathbb{Z}\right)$ is an isomorphism as well.

Since the cycle class map commutes with Gysin maps [Voi07, Proposition 9.2.1(ii)], there is a commutative diagram of the form

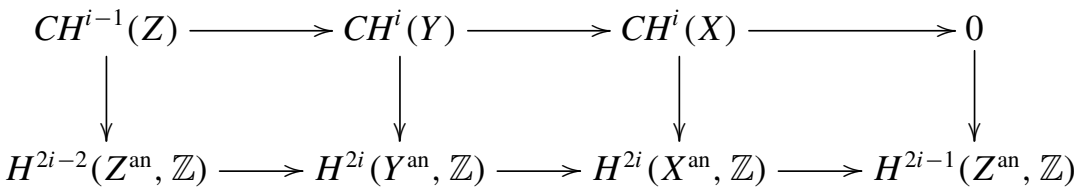

The map $c l: C H^{i-1}(Z) \rightarrow H^{2 i-2}\left(Z^{\text {an }}, \mathbb{Z}\right)$ is an isomorphism for $i=1$ essentially by definition and an isomorphism for $i=2$ by the discussion of the previous paragraph. The map $c l: C H^{i}(Y) \rightarrow H^{2 i}\left(Y^{\text {an }}, \mathbb{Z}\right)$ is an isomorphism for $i \leqslant 2$ by assumption. Since the right vertical map is injective, it follows from the five lemma that $C H^{i}(X) \rightarrow H^{2 i}\left(X^{\text {an }}, \mathbb{Z}\right)$ is injective for $i \leqslant 2$ as well.

REMARK 3.1.2. If $Y$ is 1-connected, it follows from the Lefschetz theorem on the fundamental group (see, for example, [Har70, Corollary IV.2.2]) that $Z$ is also 1-connected. In that case, one concludes that $\operatorname{Pic}(X) \rightarrow H^{2}\left(X^{\text {an }}, \mathbb{Z}\right)$ is an isomorphism as well since $H^{1}\left(Z^{\text {an }}, \mathbb{Z}\right)=0$ by the Hurewicz theorem. One way to guarantee that $Y$ is simply connected and that the cycle class map $\mathrm{CH}^{i}(Y) \rightarrow$ $H^{2 i}\left(Y^{\text {an }}, \mathbb{Z}\right)$ is an isomorphism is to require that $Y$ admit a cellular decomposition in the sense of, for example, [Ful98, Example 1.9.1]. Recall that a variety $Y$ is said to admit a cellular decomposition if it admits an increasing filtration by closed subvarieties $\emptyset=Y_{-1} \subset \cdots \subset Y_{i} \subset Y_{i+1} \subset \cdots \subset Y_{n}=Y$ such that $Y_{i} \backslash Y_{i-1}$ can be written as a disjoint union of varieties isomorphic to affine space. In that case, 
$Y$ is a rational variety and $Y^{\text {an }}$ is simply connected, for example, by [Ser59]. In the examples we consider below, $Y$ will admit a cellular decomposition.

If $Y$ admits a cellular decomposition, then the cohomology of $Y^{\text {an }}$ is concentrated in even degrees. In particular, $H^{5}\left(Y^{\text {an }}, \mathbb{Z}\right)=0$ and therefore the map $H^{4}\left(X^{\text {an }}, \mathbb{Z}\right) \rightarrow H^{3}\left(Z^{\text {an }}, \mathbb{Z}\right)$ in the Gysin sequence is surjective. By taking $Z$ to be a sufficiently ample hypersurface, we can guarantee that $H^{3}\left(Z^{\text {an }}\right.$, $\mathbb{Z}$ ) is nonzero in general (for example, take a smooth hypersurface of degree $\geqslant 5$ in $\left.\mathbb{P}^{4}\right)$. Thus, in general, $\mathrm{CH}^{2}(X) \rightarrow H^{4}\left(X^{\text {an }}, \mathbb{Z}\right)$ need not be surjective.

Fix an isomorphism $C H^{*}\left(\mathbb{P}^{1} \times \mathbb{P}^{3}\right) \cong \mathbb{Z}[\xi, \tau] /\left\langle\xi^{2}, \mu^{4}\right\rangle$ (here $\xi$ and $\mu$ are elements of degree one in the Chow ring). If $Z$ is a smooth hypersurface of bidegree $\left(d_{1}, d_{2}\right)$, then under this isomorphism $[Z]=d_{1} \xi+d_{2} \mu$. We now identify the Chow groups of the complement of $Z$ in $\mathbb{P}^{1} \times \mathbb{P}^{3}$ in low degrees; we do this since the computation is concrete and elementary (the full strength of this statement is not necessary in Theorem 3.1.4 below).

Proposition 3.1.3. Suppose $Z \subset \mathbb{P}^{1} \times \mathbb{P}^{3}$ is a hypersurface of bidegree $\left(d_{1}, d_{2}\right)$ with $d_{1} \neq 0, d_{2} \neq 0$. Set $g=\operatorname{gcd}\left(d_{1}, d_{2}\right)$ and pick $m$ and $n$ such that $m d_{1}+n d_{2}=g$. If $X:=\mathbb{P}^{1} \times \mathbb{P}^{3} \backslash Z$, then

$$
\begin{aligned}
& C H^{1}(X) \cong \mathbb{Z} / d_{1} \mathbb{Z} \oplus \mathbb{Z} / d_{2} \mathbb{Z}, \text { and } \\
& C H^{2}(X) \cong \mathbb{Z} / g \mathbb{Z} \oplus \mathbb{Z} / \frac{d_{2}^{2}}{g} \mathbb{Z} .
\end{aligned}
$$

Under the first isomorphism $\mathbb{Z} / d_{1} \mathbb{Z}$ is generated by the image of $\xi$ while $\mathbb{Z} / d_{2} \mathbb{Z}$ is generated by the image of $\mu$. Under the second isomorphism, the class $\xi \mu$ is sent to $\left(1,-m d_{2} / g\right)$, while the class $\mu^{2}$ is sent to a generator of $\mathbb{Z} /\left(d_{2}^{2} / g\right) \mathbb{Z}$. If $d_{1} \nmid d_{2}$ and $d_{2} \nmid d_{1}$ (for example, if $\operatorname{gcd}\left(d_{1}, d_{2}\right)=1$ ), then $\xi \mu$ can be assumed to restrict nontrivially to $\mathrm{CH}^{2}(\mathrm{X})$.

Proof. It suffices to identify the map $C H^{j-1}(Z) \rightarrow C H^{j}\left(\mathbb{P}^{1} \times \mathbb{P}^{3}\right)$. Since $i^{*}$ : $C H^{j-1}\left(\mathbb{P}^{1} \times \mathbb{P}^{3}\right) \rightarrow C H^{j-1}(Z)$ is an isomorphism for $j \leqslant 2$ as observed in the proof of Proposition 3.1.1, it remains to compute the cokernel of the map $i^{*} i_{*}$ : $C H^{j-1}\left(\mathbb{P}^{1} \times \mathbb{P}^{3}\right) \rightarrow C H^{j}\left(\mathbb{P}^{1} \times \mathbb{P}^{3}\right)$, which comes from the intersection with a divisor formula [Ful98, Proposition 2.6c].

By definition, $[Z] \in C H^{1}\left(\mathbb{P}^{1} \times \mathbb{P}^{3}\right)$ is precisely $d_{1} \xi+d_{2} \mu$. The first isomorphism of the statement then follows immediately from this: $C H^{1}(X) \cong \mathbb{Z} \boxminus \oplus \mathbb{Z} \mu /\left\langle d_{1} \xi+\right.$ $\left.d_{2} \mu\right\rangle$. For the second isomorphism we proceed as follows. Consider the matrix representing $i^{*} i_{*}$ with respect to the bases $\xi, \mu$ of $C H^{1}\left(\mathbb{P}^{1} \times \mathbb{P}^{3}\right)$ and $\xi \mu, \mu^{2}$ of $C H^{2}\left(\mathbb{P}^{1} \times \mathbb{P}^{3}\right)$. This matrix is not diagonal, but can be put in Smith normal form 
via the identity:

$$
\left(\begin{array}{cc}
1 & 0 \\
-\frac{m d_{2}}{g} & 1
\end{array}\right)\left(\begin{array}{ll}
d_{2} & d_{1} \\
0 & d_{2}
\end{array}\right)\left(\begin{array}{cc}
n & -\frac{d_{1}}{g} \\
m & \frac{d_{2}}{g}
\end{array}\right)=\left(\begin{array}{ll}
g & 0 \\
0 & \frac{d_{2}^{2}}{g}
\end{array}\right) .
$$

In particular, the cokernel of the map $i^{*} i_{*}$ can be computed from that of $\operatorname{diag}(g$, $d_{2}^{2} / g$ ), which is what was asserted in the statement. The statements regarding the images of $\xi \mu$ and $\mu^{2}$ are immediate, and the final statement follows from the choice of Bézout identity presenting $\operatorname{gcd}\left(d_{1}, d_{2}\right)$ that arises from the Euclidean algorithm.

THEOREM 3.1.4. Suppose $Z \subset \mathbb{P}^{1} \times \mathbb{P}^{3}$ is a smooth complex hypersurface of bidegree $(3,4)$ defined over $\overline{\mathbb{Q}}$ that specializes modulo some prime $p$ to the singular hypersurface $y_{0}^{3} x_{0}^{4}+y_{0}^{2} y_{1} x_{1}^{4}+y_{0} y_{1}^{2} x_{2}^{4}+y_{1}^{3} x_{3}^{4}$ over $\overline{\mathbb{F}}_{p}$. The classes $\xi \mu$ and $\xi \mu^{2}$ both restrict nontrivially from $\mathbb{P}^{1} \times \mathbb{P}^{3}$ to $X=\left(\mathbb{P}^{1} \times \mathbb{P}^{3}\right) \backslash Z$ and if $\psi$ is the image of $\xi \mu \in C H^{2}(X)$, then $S q^{2} \psi \neq 0 \in C H^{3}(X) / 2$.

Proof. Observe that $S q^{2}(\xi \mu)=\xi \mu^{2}+\xi^{2} \mu=\xi \mu^{2}$ in $C H^{*}\left(\mathbb{P}^{1} \times \mathbb{P}^{3}\right)$ by the Cartan formula [Voe03, Proposition 9.7] (note that $S q^{1}(\xi)=S q^{1}(\mu)=0$ since $H^{3,1}(W$, $\mathbb{Z})$ vanishes for any smooth scheme $W$ ). Since motivic Steenrod operations are compatible with pullbacks along morphisms of smooth schemes by construction, the statement that $S q^{2}(\psi)$ is nontrivial will follow from the assertion that $\xi \mu$ and $\xi \mu^{2}$ restrict nontrivially to $X$. Since $\operatorname{gcd}(3,4)=1$, the fact that $\xi \mu$ restricts nontrivially to $X$ follows immediately from Proposition 3.1.3. Thus, it remains to show that $\xi \mu^{2}$ restricts nontrivially to $X$.

To this end, we use an idea of Totaro who showed that every curve in $Z$ has even degree over $\mathbb{P}^{1}$. Indeed, see [Tot13, Proof of Theorem 3.1] for this precise statement. Given this statement, one deduces immediately that $i_{*}[C]$ for a 1-cycle $C$ on $Z$ is of the form $2 r \xi \mu^{2}+s \mu^{3}$. In particular, $\xi \mu^{2}$ does not lie in the image of $i_{*}$ or the corresponding map for Chow groups modulo 2 and therefore restricts nontrivially to $C H^{3}(X) / 2$. (Note: this last observation reproves the fact that $\xi \mu$ restricts nontrivially to $X$ since it follows immediately from the previous statement that $S q^{2}(\psi)$, which is the restriction of $S q^{2}(\xi \mu)=\xi \mu^{2}$ to $X$, is nontrivial.)

COROllary 3.1.5. Suppose $X$ is a variety as described in the statement of Theorem 3.1.4. For a given $c_{2}^{\text {top }} \in H^{4}\left(X^{\text {an }}, \mathbb{Z}\right)$, there is a unique topological vector bundle $\mathcal{E}^{\mathrm{an}}$ over $X^{\mathrm{an}}$ with $c_{1}^{\text {top }}\left(\mathcal{E}^{\mathrm{an}}\right)=0$ and $c_{2}^{\text {top }}\left(\mathcal{E}^{\mathrm{an}}\right)=c_{2}^{\text {top }}$. If $c_{2}^{\text {top }}$ is the image 
of $\xi \mu$ under the cycle class map, then the topological vector bundle with Chern classes $\left(0, c_{2}^{\text {top }}\right)$ has algebraic Chern classes yet fails to be algebraizable.

Proof. As discussed in the proof of Theorem 2 at the end of Subsection 2.2 the map $\left(c_{1}^{\text {top }}, c_{2}^{\text {top }}\right): \mathscr{V}_{2}^{\text {top }}(X) \rightarrow H^{2}\left(X^{\text {an }}, \mathbb{Z}\right) \times H^{4}\left(X^{\text {an }}, \mathbb{Z}\right)$ is a pointed bijection. Thus, the pair $\left(0, c_{2}^{\text {top }}\right)$ determines a unique rank-two topological vector bundle on $X^{\text {an }}$. In the case where $c_{2}^{\text {top }}$ is the image of $\xi \mu$ under restriction, it follows immediately from Theorem 3.1.4 that $S q^{2}(\xi \mu) \neq 0 \in C H^{3}(X) / 2$. Therefore, the claim follows from Theorem 2.2.2.

3.2. Conjectures on cycles and genericity of the examples. The example of the previous section seems intimately related to the failure of the integral Hodge conjecture. We explore this connection in greater detail now.

EXAMPLE 3.2.1. There are examples due to Kollár-van Geemen that show the integral Hodge conjecture can fail for hypersurfaces in projective space; see [Tre92] or [SV05, Theorem 2]. Indeed, suppose $Z \subset \mathbb{P}^{4}$ is a hypersurface of degree $d$. Assume that for some integer $p$ coprime to $6, p^{3}$ divides $d$. Then, for a general $Z$, any curve $C \subset Z$ has degree divisible by $p$. Observe that, in this case, $\operatorname{Pic}(Z) \cong \mathbb{Z}$ by the Grothendieck-Lefschetz theorem. Setting $X:=\mathbb{P}^{4} \backslash Z$, we can compute $C H^{i}(X)$ in low degrees. Identify $C H^{*}\left(\mathbb{P}^{4}\right)=\mathbb{Z}[\xi] / \xi^{5}$. Then, $C H^{1}(X)=\mathbb{Z} / d \mathbb{Z}$ generated by the image of $\xi$ and $C H^{2}(X)=\mathbb{Z} / d \mathbb{Z}$ generated by the class of $\xi^{2}$. Note that $S q^{2}\left(\xi^{2}\right)=2 \xi S q^{2} \xi=0 \in C H^{3}(X) / 2$. Take a topological complex vector bundle on $X$ of rank two with Chern classes of the form $(m \xi$, $a \xi^{2}$ ). If $m$ is even, all such bundles are necessarily algebraizable. If $m$ is odd, then $S q^{2}\left(a \xi^{2}\right)+m \xi \cup a \xi^{2}=a m \xi^{3}$. If $a$ is also odd, then $a m$ is odd and so $a m \xi^{3}$ could restrict nontrivially to $C H^{3}(X) / 2$. However, the construction above only shows that $C H^{3}(X)$ is a quotient of $\mathbb{Z} / n \mathbb{Z}$, where $p \mid n$. However, since $p$ is odd, we do not know whether $\xi^{3}$ restricts nontrivially to $C H^{3}(X) / 2$.

EXAMPLE 3.2.2. By [Tot13, Lemma 5.1], there is a smooth hypersurface $Z \subset \mathbb{P}^{4}$ of degree 48 over $\overline{\mathbb{Q}}$ for which the integral Hodge conjecture fails. The construction of this example is, however, rather involved. Set $X=\mathbb{P}^{4} \backslash Z$. In this case, $\operatorname{Pic}(X) \cong \mathbb{Z} / 48 \mathbb{Z}$ and $C H^{2}(X) \cong \mathbb{Z} / 48 \mathbb{Z}$, generated by $\xi$ and $\xi^{2}$ in the notation of Example 3.2.1. Totaro shows that every curve $C \subset X$ has even degree over $\overline{\mathbb{Q}}$, that is, the pushforward map $\mathrm{CH}^{2}(Z) \rightarrow C H^{3}(X)$ has image contained in $2 \xi^{3}$. In particular, in this example $C H^{3}(X) / 2$ is necessarily nontrivial, and $\xi^{2}$ restricts nontrivially. As before, if we fix Chern classes $\left(m \xi, a \xi^{2}\right)$ with both $a$ and $m$ odd, then $m a \xi^{3}$ restricts nontrivially to $C H^{3}(X) / 2$ and we thus obtain more nonalgebraizable bundles with algebraic Chern classes. 
Let us recall the following conjecture of Nori, as modified by Totaro.

CONJECTURE 3.2 .3 (Nori,Totaro). If $Y$ is a smooth projective variety, and $Z \subset Y$ is a very general, sufficiently ample hypersurface, then the restriction map $\mathrm{CH}^{i}(Y) \rightarrow C H^{i}(Z)$ is an isomorphism for $i<\operatorname{dim} Z$.

Combined with Theorem 2, Conjecture 3.2.3 suggests that nonalgebraizable topological complex vector bundles of rank two should be rather common.

EXAMPLE 3.2.4. Under the hypotheses of Conjecture 3.2.3, set $\xi=[Z] \in \operatorname{Pic}(Y)$ and $X:=Y \backslash Z$, which is necessarily affine. Observe then that if $Z \subset Y$ has dimension three, then $\operatorname{Pic}(X)=\operatorname{Pic}(Y) /\langle\xi\rangle, C H^{2}(X)=C H^{2}(Y) /\langle\xi \cup \operatorname{Pic}(Y)\rangle$, while $C H^{3}(X)=C H^{3}(Y) /\left\langle\xi \cup C H^{2}(Y)\right\rangle$. Thus, assuming Nori's conjecture, if $Y=\mathbb{P}^{4}$ then for $d$ sufficiently large, and any sufficiently general hypersurface of degree $d$, then $\operatorname{Pic}(X) \cong \mathbb{Z} / d \mathbb{Z}, C H^{2}(X) \cong \mathbb{Z} / d \mathbb{Z}$ and $C H^{3}(X) \cong \mathbb{Z} / d \mathbb{Z}$ generated by the image of $\xi, \xi^{2}, \xi^{3}$. If $d$ is even, then we expect only those topological complex vector bundles of rank two whose Chern classes are of the form $\left(m \xi, a \xi^{2}\right)$ with $a$ and $m$ both odd to be nonalgebraizable.

\section{Acknowledgements}

The first author would like to thank Burt Totaro for some helpful correspondence as regards the integral Hodge conjecture on 3-folds and for pointing out the paper [Tot13]. Asok was supported by National Science Foundation Award DMS-1254892. The third author would like to thank Philip Griffiths for correspondence related to the paper [Gri72], which was a source of inspiration for the questions considered here. Hopkins was supported by National Science Foundation Award DMS-0906194. Finally, the authors thank Oliver Röndigs for some comments on the first version of this paper, and the referees for some helpful comments and questions.

\section{References}

[AF14a] A. Asok and J. Fasel, 'Algebraic vector bundles on spheres', J. Topol. 7(3) (2014), 894-926.

[AF14b] A. Asok and J. Fasel, 'A cohomological classification of vector bundles on smooth affine threefolds', Duke Math. J. 163(14) (2014), 2561-2601.

[AF15a] A. Asok and J. Fasel, 'Secondary characteristic classes and the Euler class', Doc. Math. (2015), 7-29. (Extra vol.: Alexander S. Merkurjev's Sixtieth Birthday).

[AF15b] A. Asok and J. Fasel, 'Splitting vector bundles outside the stable range and homotopy theory of punctured affine spaces', J. Amer. Math. Soc. 28(4) (2015), 1031-1062. 
[AF17] A. Asok and J. Fasel, 'An explicit $K O$-degree map and applications', J. Topol. 10(1) (2017), 268-300.

[AHW17] A. Asok, M. Hoyois and M. Wendt, 'Affine representability results in $\mathbb{A}^{1}$-homotopy theory I: vector bundles', Duke Math. J. 166(10) (2017), 1923-1953.

[AR76] M. F. Atiyah and E. Rees, 'Vector bundles on projective 3-space', Invent. Math. 35 (1976), 131-153.

[BP87] C. Bănică and M. Putinar, 'On complex vector bundles on projective threefolds', Invent. Math. 88(2) (1987), 427-438.

[Bro03] P. Brosnan, 'Steenrod operations in Chow theory', Trans. Amer. Math. Soc. 355(5) (2003), 1869-1903 (electronic).

[Del09] P. Deligne, 'Voevodsky's lectures on motivic cohomology 2000/2001', in Algebraic Topology, Abel Symp., 4 (Springer, Berlin, 2009), 355-409.

[DI05] D. Dugger and D. C. Isaksen, 'Motivic cell structures', Algebr. Geom. Topol. 5 (2005), 615-652.

[EG98] D. Edidin and W. Graham, 'Equivariant intersection theory', Invent. Math. 131(3) (1998), 595-634.

[Fas13] J. Fasel, 'The projective bundle theorem for $\mathbf{I}^{j}$-cohomology', J. K-Theory $\mathbf{I}(2)$ (2013), 413-464.

[For11] F. Forstnerič, Stein Manifolds and Holomorphic Mappings, Ergebnisse der Mathematik und ihrer Grenzgebiete. 3. Folge. A Series of Modern Surveys in Mathematics, 56 (Springer, Heidelberg, 2011).

[Fu198] W. Fulton, Intersection Theory, 2nd edn, Ergebnisse der Mathematik und ihrer Grenzgebiete. 3. Folge. A Series of Modern Surveys in Mathematics, 2 (Springer, Berlin, 1998).

[Gil81] H. Gillet, 'Riemann-Roch theorems for higher algebraic $K$-theory', Adv. Math. 40(3) (1981), 203-289.

[Gra58] H. Grauert, 'Analytische Faserungen über holomorph-vollständigen Räumen', Math. Ann. 135 (1958), 263-273.

[Gri72] P. A. Griffiths, 'Function theory of finite order on algebraic varieties. i(a)', $J$. Differential Geometry 6 (1971/72), 285-306.

[Har70] R. Hartshorne, Ample Subvarieties of Algebraic Varieties, Lecture Notes in Mathematics, 156 (Springer, Berlin-New York, 1970), Notes written in collaboration with C. Musili.

[KM82] N. M. Kumar and M. P. Murthy, 'Algebraic cycles and vector bundles over affine threefolds', Ann. of Math. (2) 116(3) (1982), 579-591.

[MVW06] C. Mazza, V. Voevodsky and C. Weibel, Lecture Notes on Motivic Cohomology, Clay Mathematics Monographs, 2 (American Mathematical Society, Providence, RI, 2006).

[Mor04] F. Morel, 'Sur les puissances de l'idéal fondamental de l'anneau de Witt', Comment. Math. Helv. 79(4) (2004), 689-703.

[Mor12] F. Morel, $\mathbb{A}^{1}$-Algebraic Topology Over a Field, Lecture Notes in Mathematics, 2052 (Springer, Heidelberg, 2012).

[MV99] F. Morel and V. Voevodsky, 'A $\mathbb{1}^{1}$-homotopy theory of schemes', Publ. Math. Inst. Hautes Études Sci. 90(2001) (1999), 45-143.

[MS76] M. P. Murthy and R. G. Swan, 'Vector bundles over affine surfaces', Invent. Math. 36 (1976), 125-165.

[OVV07] D. Orlov, A. Vishik and V. Voevodsky, 'An exact sequence for $K_{*}^{M} / 2$ with applications to quadratic forms', Ann. of Math. (2) 165(1) (2007), 1-13. 
[Pus04] O. Pushin, 'Higher Chern classes and Steenrod operations in motivic cohomology', J. K-Theory 31(4) (2004), 307-321.

[Sch61] R. L. E. Schwarzenberger, 'Vector bundles on algebraic surfaces', Proc. Lond. Math. Soc. (3) 11 (1961), 601-622.

[Ser58] J.-P. Serre, 'Modules projectifs et espaces fibrés à fibre vectorielle', in Séminaire P. Dubreil, M.-L. Dubreil-Jacotin et C. Pisot, 1957/58, Fasc. 2, Exposé 23 (Secrétariat mathématique, Paris, 1958), 18.

[Ser59] J.-P. Serre, 'On the fundamental group of a unirational variety', J. Lond. Math. Soc. 34 (1959), 481-484.

[Sus87] A. A. Suslin, 'Torsion in $K_{2}$ of fields', $K$-Theory $1(1)$ (1987), 5-29.

[SV05] C. Soulé and C. Voisin, 'Torsion cohomology classes and algebraic cycles on complex projective manifolds', Adv. Math. 198(1) (2005), 107-127.

[Tot03] B. Totaro, 'Non-injectivity of the map from the Witt group of a variety to the Witt group of its function field', J. Inst. Math. Jussieu 2(3) (2003), 483-493.

[Tot13] B. Totaro, 'On the integral Hodge and Tate conjectures over a number field', Forum Math. Sigma 1 (2013), e4, 13pp.

[Tre92] Trento examples, Classification of Irregular Varieties (Trento 1990), Lecture Notes in Mathematics, 1515 (Springer, Berlin, 1992), 134-139.

[Voe03] V. Voevodsky, 'Reduced power operations in motivic cohomology', Publ. Math. Inst. Hautes Études Sci. 98 (2003), 1-57.

[Voe04] V. Voevodsky, 'On the zero slice of the sphere spectrum', Tr. Mat. Inst. Steklova 246(Algebr. Geom. Metody, Svyazi i Prilozh) (2004), 106-115.

[Voi07] C. Voisin, Hodge Theory and Complex Algebraic Geometry. II, English edn, Cambridge Studies in Advanced Mathematics, 77 (Cambridge University Press, Cambridge, 2007), Translated from the French by Leila Schneps. 